\title{
Color Fusion of Magnetic Resonance Images Improves Intracranial Volume Measurement in Studies of Aging
}

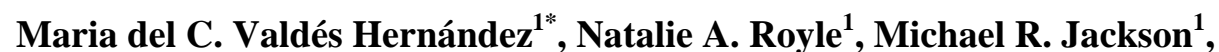 \\ Susana Muñoz Maniega ${ }^{1}$, Lars Penke ${ }^{2}$, Mark E. Bastin ${ }^{3}$, Ian J. Deary ${ }^{2}$, Joanna M. Wardlaw ${ }^{1}$ \\ ${ }^{1}$ Brain Research Imaging Centre, University of Edinburgh, Edinburgh, UK \\ ${ }^{2}$ Department of Psychology, University of Edinburgh, Edinburgh, UK \\ ${ }^{3}$ Department of Medical and Radiological Sciences, University of Edinburgh, Edinburgh, UK \\ Email: "mvhernan@staffmail.ed.ac.uk
}

Received December 22, 2011; revised January 24, 2012; accepted February 2, 2012

\begin{abstract}
Background: Comparison of intracranial volume (ICV) measurements in different subpopulations offers insight into age-related atrophic change and pathological loss of neuronal tissue. For such comparisons to be meaningful the accuracy of ICV measurement is paramount. Color magnetic resonance images (MRI) have been utilised in several research applications and are reported to show promise in the clinical arena. Methods: We selected a sample of 150 older community-dwelling individuals (age 71 to 72 years) representing a wide range of ICV, white matter lesions and atrophy. We compared the extraction of ICV by thresholding on T2*-weighted MR images followed by manual editing (reference standard) done by an analyst trained in brain anatomy, with thresholding plus computational morphological operations followed by manual editing on a framework of a color fusion technique (MCMxxxVI) and two automatic brain segmentation methods widely used, these last three done by two image analysts. Results: The range of ICV was 1074 to $1921 \mathrm{~cm}^{3}$ for the reference standard. The mean difference between the reference standard and the ICV measured using the technique that involved the color fusion was $2.7 \%$, while it was $5.4 \%$ compared with any fully automatic technique. However, the $95 \%$ confidence interval of the difference between the reference standard and each method was similar: it was $7 \%$ for the segmentation aided by the color fusion and was $7 \%$ and $8.3 \%$ for the two fully automatic methods tested. Conclusion: For studies of aging, the use of color fusion MRI in ICV segmentation in a semi-automatic framework delivered best results compared with a reference standard manual method. Fully automated methods, while fast, all require manual editing to avoid significant errors and, in this post-processing step color fusion MRI is recommended.
\end{abstract}

Keywords: Intracranial Volume; Segmentation; Brain Volume; Aging; Color MRI

\section{Introduction}

Brain size, assessed volumetrically, varies between people and changes over the lifespan [1-4]. Age-related atrophy occurs in healthy older adults, with limited data available to date showing that grey matter volume declines steadily with age and white matter volume begins to decline after middle-age [5]. Reduction in brain size over the lifespan has been associated with a general decline in cognitive performance in normal healthy older adults $[6$, 7].

To accurately assess the degree to which age-related atrophy is associated with cognitive decline in healthy aging, baseline measures of both cognitive ability and brain size are required. In young adults the brain normally fits strongly inside the inner table of the skull, therefore the intracranial volume (ICV) is considered

"Corresponding author. approximately the same as the maximum mature brain size [8]. But in individuals of increasing maturity, loss of brain tissue means that a higher proportion of ICV represents cerebrospinal fluid (CSF), either within the ventricles or in the extra-axial spaces. Thus ICV offers a potentially reproducible measure of maximum adult brain size in any given individual.

As methods to limit the adverse effects of aging and brain research associated with that, are an increasing priority for many governments, validated methods of measuring ICV quickly and reliably for use in large imaging studies of aging are required. A variety of different methods of calculating ICV have been suggested. However, these methods of estimating ICV have generally not been tested for accuracy or reliability [8]. ICV can be estimated by measuring the intracranial cross-sectional area (ICA) from a midline sagittal image, which is quicker than full ICV measurement $[9,10]$. Full ICV measurement 
traditionally requires manual tracing around, or thresholding on, the boundary between the inner skull/CSF interface (assuming that the dural thickness is negligible) on each slice on which the ICV is visible. However this is very time consuming and subject to observer bias. Thus, some studies have used a brain volume extracted using fully automated methods as a surrogate for ICV $[11,12]$, and several such techniques have been described [13]. However, studies of automatic brain extraction methods have shown that editing of extracranial structures is required to accurately extract brain volume [14]. This paper presents an alternative method for removing extracranial tissue in a semi-automated way, combining images from more than one MRI pulse sequence to produce a color image that combines attributes from each of the original sequences.

This concept is not new. Color MR images were suggested by Holland and Bottomley more than thirty years ago [15], and the first clinical color MR images were published in 1987 by Weiss et al. [16]. Soon after, different color composite techniques were implemented in different ways to improve the information content and enhance conspicuity of specific tissues and fluids (Table 1).
After almost a decade, in 2009, the RSNA unveiled a new color MRI software package: Rev ColorMRI (http:// www.revolutionsmedical.com/RevMed-ColorMRI.php) and a year later, Valdes Hernandez et al. published a technique that fuses two different MR sequences mapped in the red-green space to segment brain tissues and white matter lesions: Multispectral Color Modulation and Variance Identification (MCMxxxVI) [17], and made it freely available as an open source product (http://sourceforge. net/projects/bric1936/). We applied this technique to ICV segmentation and evaluated its results, comparing them with well-established segmentation techniques widely used in clinical research [13].

\section{Materials and Methods}

\subsection{Subjects}

We selected 150 subjects (71 - 72 years old; 87 males and 63 females) from the Lothian Birth Cohort 1936 (LBC1936) [18]. This cohort includes 1091 relatively healthy community dwelling older subjects born in 1936 who took part in the Scottish Mental Health Survey in 1947 (Moray House Test), a validated measure of cognitive ability, thus providing early life cognitive data.

Table 1. Main features of the color MRI techniques developed after the first color images were published in 1987.

\begin{tabular}{|c|c|c|c|}
\hline Paper & Name of the technique & Principles & Advantages/disadvantages \\
\hline $\begin{array}{l}\text { Weiss K.L. et al. } \\
\quad 1987[16]\end{array}$ & Hybrid color MRI & $\begin{array}{l}\text { Combines and displays data from two different MR } \\
\text { sequences of the same anatomic slice in a single } \\
\text { color image. } \\
\text { Varying spectral hues are assigned to pixel } \\
\text { intensities from one image. } \\
\text { The luminance is derived from the intensities of the } \\
\text { corresponding pixels of a second spatially aligned } \\
\text { image. }\end{array}$ & $\begin{array}{l}\text { Produces images of improved information } \\
\text { content. } \\
\text { Number of luminance levels was } 4 \text {. Further } \\
\text { work needed to increase it to } 8 \text { or } 16 \text {. }\end{array}$ \\
\hline $\begin{array}{l}\text { Wells, M.G. et al. } \\
\quad 1989 \text { [25] }\end{array}$ & Color composite & $\begin{array}{l}\text { Combines information from two images } \\
\text { (T1-weighted and PD) such that the values from one } \\
\text { image are represented by a change in hue (color), } \\
\text { and the values from the other by a change in } \\
\text { luminance (intensity). }\end{array}$ & $\begin{array}{l}\text { For the majority of cases the combined } \\
\text { image can effectively represent the } \\
\text { information normally contained in both } \\
\text { images-thus speeding up the viewing } \\
\text { process. }\end{array}$ \\
\hline $\begin{array}{l}\text { Kamman R.L. et al. } \\
1989 \text { [27] }\end{array}$ & Color composite & $\begin{array}{l}\text { Color images are computed from } \mathrm{T} 1 \text { and } \mathrm{T} 2 \text { using } \\
\text { matrix multiplication on a pixel base. } \\
\text { Color resolution could be modified using different } \\
\text { choices of the reference triangle in which the color } \\
\text { combinations were defined. }\end{array}$ & $\begin{array}{l}\text { This method of representation offers a } \\
\text { means for displaying multiple features, } \\
\text { independent of instrumental settings. }\end{array}$ \\
\hline $\begin{array}{l}\text { Brown H.K. et al. } \\
1991,1992 \text { and } 1993 \\
{[23,24,28]}\end{array}$ & Color composite & $\begin{array}{l}\text { Calculates the mean intensity values on region of } \\
\text { interests (ROI) and applies various colors: one to } \\
\text { each ROI of two spatially aligned images. The tissue } \\
\text { contrast patterns are optimized in the final image. }\end{array}$ & $\begin{array}{l}\text { Generates seminatural-appearing color } \\
\text { images that possessed enhanced } \\
\text { conspicuity of specific tissues and fluids. }\end{array}$ \\
\hline $\begin{array}{l}\text { Alfano B. et al. } 1992 \\
\qquad[26]\end{array}$ & Color composite & $\begin{array}{l}\mathrm{T} 1 \text { and } \mathrm{T} 2 \text { relaxation rate maps are obtained. Each } \\
\text { parameter is represented with one or with a } \\
\text { combination of two fundamental colors (red, green, } \\
\text { blue) into a composite quantitative color image. }\end{array}$ & $\begin{array}{l}\text { Each mixture of chromatic components } \\
\text { represents a unique combination of the two } \\
\text { relaxation parameters, easily interpretable } \\
\text { by the human eye, without loss of anatomic } \\
\text { information. }\end{array}$ \\
\hline $\begin{array}{l}\text { Phillips W.E. et al. } \\
1996[22]\end{array}$ & $\begin{array}{l}\text { Multiparameter full color } \\
\text { composite }\end{array}$ & $\begin{array}{l}\text { Based on the results of pathologic correlation and } \\
\text { quantitative color image analysis, applies full color } \\
\text { composite generation techniques to multiple MR } \\
\text { images: PD, T1- and T2-weighted. }\end{array}$ & $\begin{array}{l}\text { Displays clinically important } \\
\text { neuroanatomic and neuropathologic tissues. }\end{array}$ \\
\hline
\end{tabular}


For the present analysis, the sample was selected to represent the full range of early and later life cognitive ability by using the results of the Moray-House Test taken at 11 and 70 years of age [18], brain sizes, atrophy (from none to severe) and white matter lesion load, assessed as described in [19]. All subjects gave written informed consent to participate.

\subsection{MRI Acquisition}

$\mathrm{T} 2 *$-weighted $(\mathrm{T} 2 * \mathrm{~W})$ and fluid attenuated inversion recovery (FLAIR) volumes were acquired axially using a 1.5 T GE Signa Horizon HDX clinical MRI scanner (Milwaukee, WI, USA). The $\mathrm{T} 2 * \mathrm{~W}$ imaging parameters were $\mathrm{TR}=940 \mathrm{~ms}$; TE $=15 \mathrm{~ms}, 256 \times 256$ matrix size and 80 slices with voxel size of $1 \times 1 \times 2 \mathrm{~mm}$. FLAIR was acquired with $\mathrm{TR}=9002 \mathrm{~ms} ; \mathrm{TE}=147.4 \mathrm{~ms}$ and TI $=2200 \mathrm{~ms}$ with matrix size $256 \times 256$ and 40 slices with voxel size of $1 \times 1 \times 4 \mathrm{~mm}$ with slice locations matching every other slice of the $\mathrm{T} 2 * \mathrm{~W}$ volume. FLAIR volume was interpolated to 80 slices of $2 \mathrm{~mm}$ thickness by rigid body registration to the $\mathrm{T} 2 * \mathrm{~W}$ volume using FMRIB's Linear Image Registration Tool (http://www.fmrib. ox.ac. uk/fsl/).

\subsection{Image Segmentation Methods}

\subsubsection{General Considerations for Measuring ICV}

The measurements were done by three experienced image analysts all blind to each other's measurements. Each analyst was most familiar with the particular analysis method they were applying and therefore the performance could be regarded as optimal for each technique. The reference standard ICV was measured on the axial slices of the $\mathrm{T} 2 * \mathrm{~W}$ volume, semi-automatically using Analyze 9.0 as described in [9], by an image analyst trained in brain anatomy.

We defined the inferior limit of the intracranial cavity as the axial slice which was just superior to the tip of the odontoid peg at the foramen magnum (Figure 1) and was just inferior to the inferior limits of the cerebellar tonsils. We excluded the cavernous sinuses/intracranial internal carotid arteries as they enter the intracranial cavity as these are extradural. In the reference standard ICV [9], we included the dural venous sinuses and excluded the contents of the sella turcica (as being outside the main brain cavity) although we also tested the effect of including/excluding it in the analysis because some would argue that the sella should be excluded.

\subsubsection{ICV Measurement Aided by a Color Fusion Technique}

We used the Object Extraction Tool (OET) in Analyze 9.0 which applies morphological erosion, dilation, and region growing steps in addition to thresholding, to automatically extract the contents of the intracranial cavity. We used $\mathrm{T} 2 * \mathrm{~W}$ sequence because it offers the best differentiation between CSF, brain tissue and inner skull table for computational image processing.

To generate the binary mask, we placed a seed-point in the axial slice where the orbits appear and selected the optimal threshold as the intensity value that separated the optic nerve from the rest of the brain tissue. In the extraction process, morphological dilations were repeated automatically to cover $99 \%$ of the voxels in the autotraced region on the target slice. After extraction, the "holes" in the extracted "object" were filled in and a final

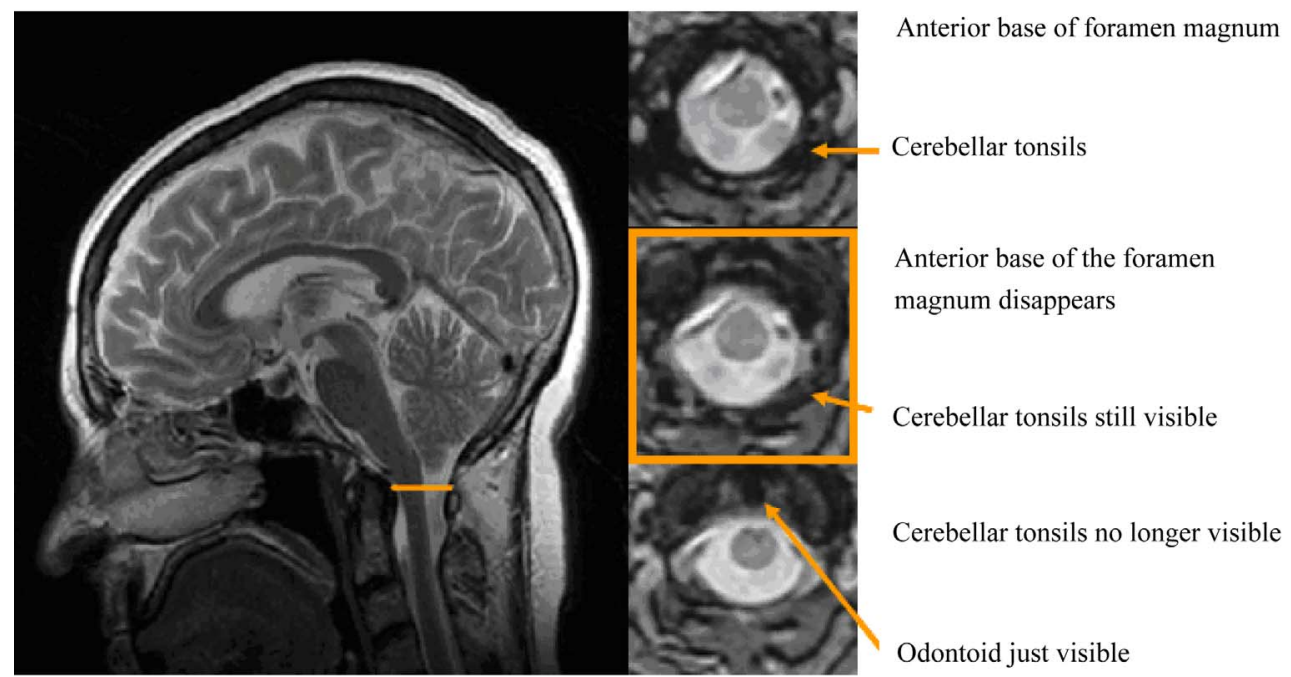

Figure 1. Midsagittal slice from a T2W MRI sequence showing how the lower limit of the intracranial volume at the foramen magnum was defined by slice at lower limit of tonsils prior to odontoid. On the right-hand an axial view of the foramen magnum (orange), including the slices superior (top) and inferior (bottom) to the slice defining the lower limit of the ICV (middle). 
6-connected 3D region growing step was performed. The removal of the extracranial tissues was done using the MCMxxxVI technique (Figure 2).

The color combination facilitates the identification of the brain boundaries in most of the controversial areas. The combination of $\mathrm{T} 2 * \mathrm{~W}$ and FLAIR in the red/green space used in [17] (Figure 2) does not show a good differentiation between the dural sinuses and the CSF. If the dural sinuses are desired to be excluded from the ICV, the modulation of T2-weighted and T1-weighted in red and green respectively is recommended (Figure 3 upper row).

The color fusion principle of MCMxxxVI is the modulation of two MR sequences using two carrier signals of wavelengths of approximately $650 \mathrm{~nm}$ and $510 \mathrm{~nm}$ respectively. For each MR sequence, the intensity level that represents the captured signal magnitude in each tissue or lesion is influenced by three parameters of the RF pulse sequence of a duration $\Delta t$ used to excite the

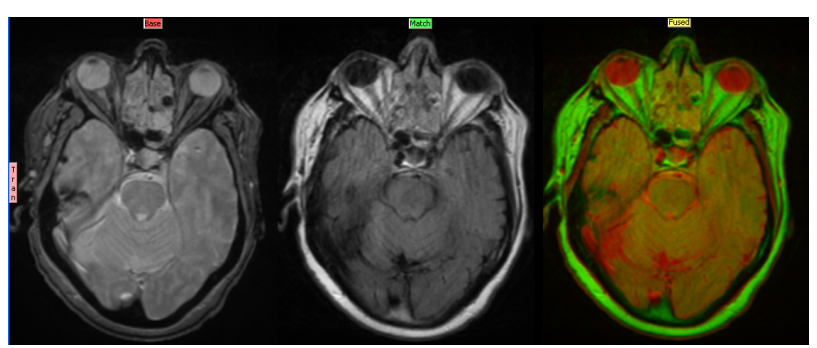

Figure 2. MCMxxxVI. Axial slice of T2*W, FLAIR and fused (red_green space) volumes of a scan showing the advantages of this technique differentiating the mucus contained in the nasal sinuses and compensating the motion artefact (colored image).

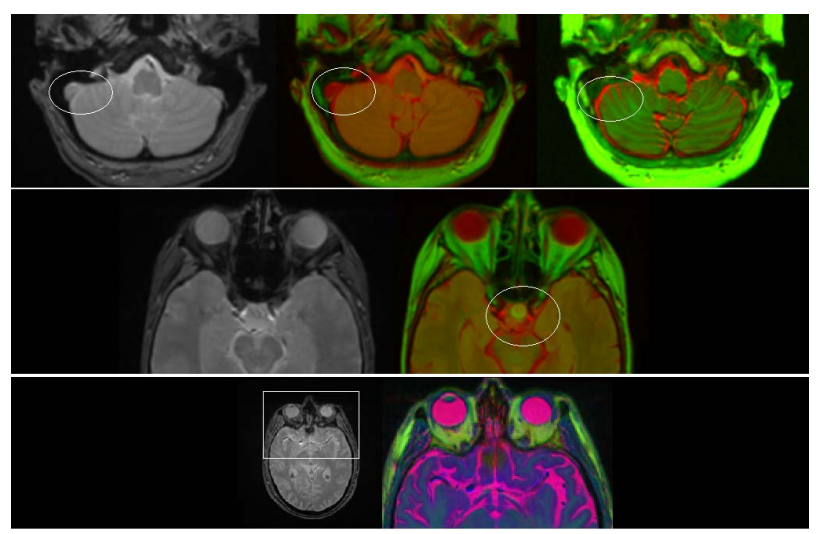

Figure 3. Controversial areas in which the color combination helps on the boundary definition. Upper row: transversal sinuses (encircled) on $\mathrm{T} 2 * \mathrm{~W}$ (left) and fusion in the red_green space of $T 2 * \mathrm{~W}$ and FLAIR (middle) and T2Wand T1W (right). Middle row: pituitary adenoma (encircled) in T2*W (left) and in the fused T2*W_FLAIR image (right). Bottom row: artefact on $\mathrm{T} 2 * \mathrm{~W}$ (left) and view of the fused $T 2 W \_T 1 W \_T 2 * W$ on the red_green_blue color space. nuclear magnetic resonance signal: the tip angle (alpha), the echo time (TE) and the pulse repetition time (TR). Therefore, for each sequence, the intensity of each voxel can be represented by a function $I$ that varies in time $t$ $(I(t))$. If a sinusoidal carrier signal has the form:

$$
A(t)=A_{0} \sin (2 \pi t f c+\phi)
$$

where:

$A(t)$ is the amplitude (or magnitude) of the signal as a function of time;

$A_{0}$ is the maximum amplitude of the signal achieved in each cycle;

$f_{c}$ is the frequency of oscillation (approximately 650 $\mathrm{nm}$ for red and $510 \mathrm{~nm}$ for green); and

$\phi$ is the phase of the signal.

When this carrier is modulated by the MR sequences, the output signal $A(t)$ will take the form:

$$
A(t)=A_{0} \sin \left(2 \pi\left[f c+\left(\Delta f / I_{0}\right) I(t)\right] t+\phi\right)
$$

$\Delta f$ delimits the range in which the output frequency can vary respect to the carrier frequency. As Equation (2) shows, now the carrier frequency term (in brackets) varies between the extremes of $f c-\Delta f$ and $f c+\Delta f$ given by the limits of variation of $I(t)$.

The software that implements this color modulation technique, afterwards reduces the numbers of colors by minimum variance quantisation and graphs the resultant colors as clusters positioned on the RGB space, thus facilitating the color discrimination of the extracranial tissues and anomalies.

This method differs from the color composite techniques summarised in Table $\mathbf{1}$ and from the one in which Rev ColorMRI is based where there is an emphasis on producing "seminatural-appearing" or "virtually realistic appearing tissue tones". While utilising a color scale that mimics the "natural appearance" of the tissues may be of benefit as an educational tool, it is unlikely that such color scale will serve to highlight pathology. Successfully differentiating otherwise unseen pathology is more likely to be achieved by the color modulation principle explained above, and will most likely be unrelated to the "natural" appearance of the tissues.

\subsubsection{Automatic Brain Extraction}

We applied two techniques: thresholding plus morphological operations using OET as explained above, but without any manual editing to obtain a surrogate ICV; and BET, from the FMRIB software library.

BET [13] performs a fully automatic brain extraction in three main steps, which is used as a surrogate measure for ICV $[11,12]$. Firstly it processes the intensity histogram to find "robust" lower and upper intensity values for the image, and a rough brain/non-brain threshold. Then, it finds the centre-of-gravity of the head image, 
along with the rough size of the head in it. Finally, it performs a triangular tessellation of a sphere's surface inside the intracranial cavity and slowly deforms it, one vertex at a time, following forces that keep the surface well-spaced and smooth, while attempting to move towards the in- tracranial cavity's edge in an iterative process. This process excludes part of the lower brain stem from the final extracted volume, although does not guarantee that its lower limit coincides with the standard boundary at the foramen magnum.

The BET fractional intensity threshold was optimized by visually inspecting the brain/ICV extractions created with a range of thresholds in a sub-sample of brain volumes. A fractional intensity threshold of 0.6 was found to be the best compromise in our sample and subsequently applied to the full dataset. We did not perform any manual editing of the derived mask obtained by this method.

\subsection{Statistical Analysis}

We compared the agreement between the volumes obtained by the following methods using Bland-Altman [20] analysis of absolute and percentage differences:

1) Reference standard vs. ICV by OET followed by MCMxxxVI, this last performed by an image analyst with minimal knowledge of brain anatomy (usual scenario)

2) Reference standard vs. "surrogate ICV" volume by BET

3) Reference standard vs. "surrogate ICV" volume by OET

4) Reference standard excluding and including the pituitary gland

Pearson correlations between methods were assessed after checking for normality using the Kolmogorov-
Smirnov test.

\section{Results}

The ICV, as measured by the reference standard method, ranged from 1074 to $1921 \mathrm{~cm}^{3}$, median $1500 \mathrm{~cm}^{3}$. The numerical values for ICV measured by each of the methods under evaluation are summarised in Table 2.

The reference standard correlated well with all other methods (Pearson's r from 0.97 to 0.98 , Table 2). The effect of including or excluding the contents of the sella turcica (mean estimated volume of this structure was approximately $2 \mathrm{~cm}^{3}$ ) were minimal, with the $95 \%$ confidence interval $(\mathrm{CI})$ of the difference in ICV calculated with and without the sella contents being $+/-0.49 \%$ from the mean $\left(7.29 \mathrm{~cm}^{3}\right)$.

Comparison of the reference standard measurements with the results obtained using MCMxxxVI, and the two fully automated methods (BET and OET) (Figure 4) showed that although the smallest mean difference was with the automatic "surrogate ICV" extracted using OET $(2.18 \%)$, this method produced the largest variability (95\% CI $+/-8.32 \%$, Table 2, Figure 4). The better performance overall was with OET followed by MCMxxxVI (mean difference $2.74 \%, 95 \% \mathrm{CI}+/-7.03 \%$ ). The largest mean difference was with 'surrogate ICV' by BET (mean difference $5.38 \%, 95 \% \mathrm{CI}+/-6.93 \%$ ).

All three automated methods produced some systematic bias albeit small in the measurement of ICV compared with the reference standard (Figure 4). The bias was least with automatic ICV extraction with OET and worst with BET. This is largely attributable to inclusion of the clivus and other extracranial structures within the ICV which occurs particularly with BET (Figure 5).

The inclusion of the cervical spinal cord below the fo-

Table 2. Comparison of the reference standard measurements with the numeric results obtained by the methods evaluated. Absolute intracranial volumes, correlation, differences in volumes with the $95 \%$ confidence intervals of the difference and \% differences (of mean ICV) between methods.

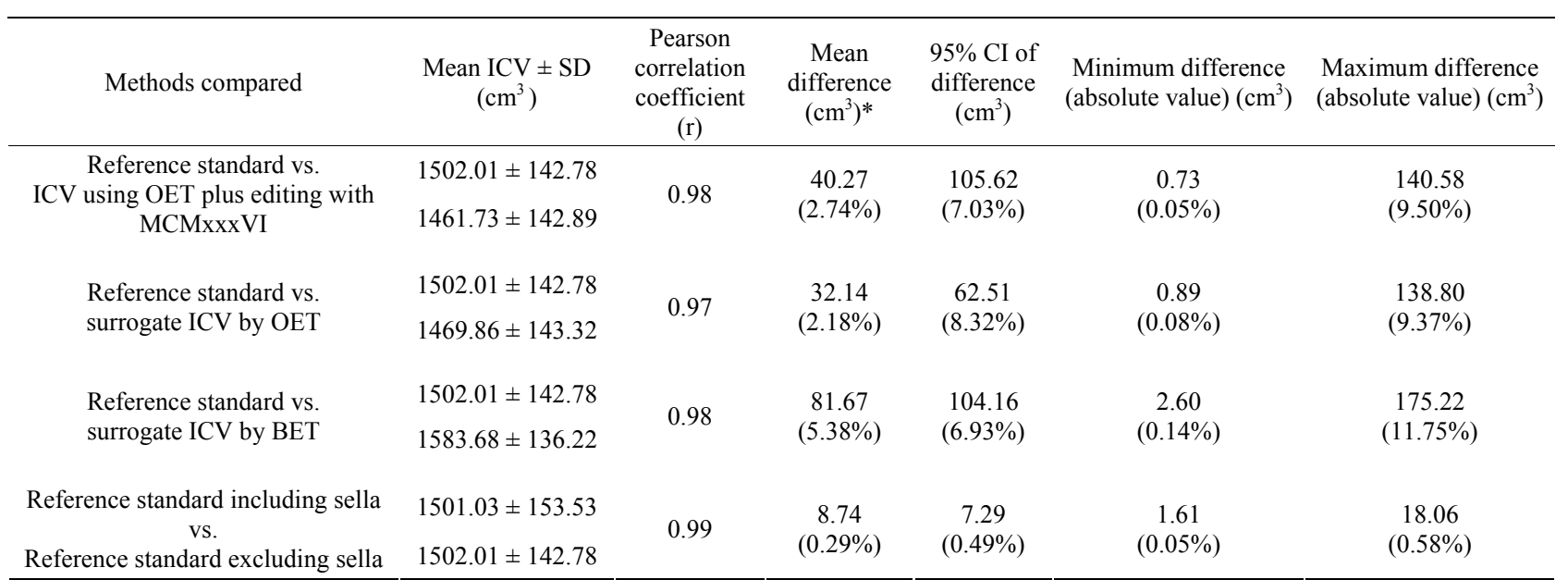

Legend: SD: standard deviation, CI: confidence interval (4SD) *\% differences from the averaged measurement between the two methods being compared. 


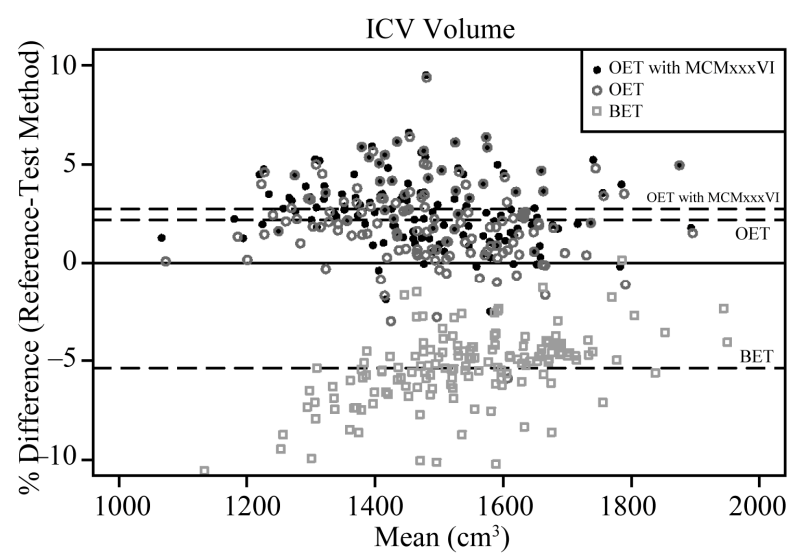

Figure 4. Bland-Altman plot comparing the ICV measurements done by thresholding followed by manual editing (reference standard) with the ICV measurements done by thresholding combined with morphological operations using Object Extraction tool in Analyze ${ }^{\mathrm{TM}}$ and manual editing in MCMxxxVI, and two different automatic techniques (Object Extraction Tool in Analyze ${ }^{\mathrm{TM}}$ and Brain Extraction Tool).

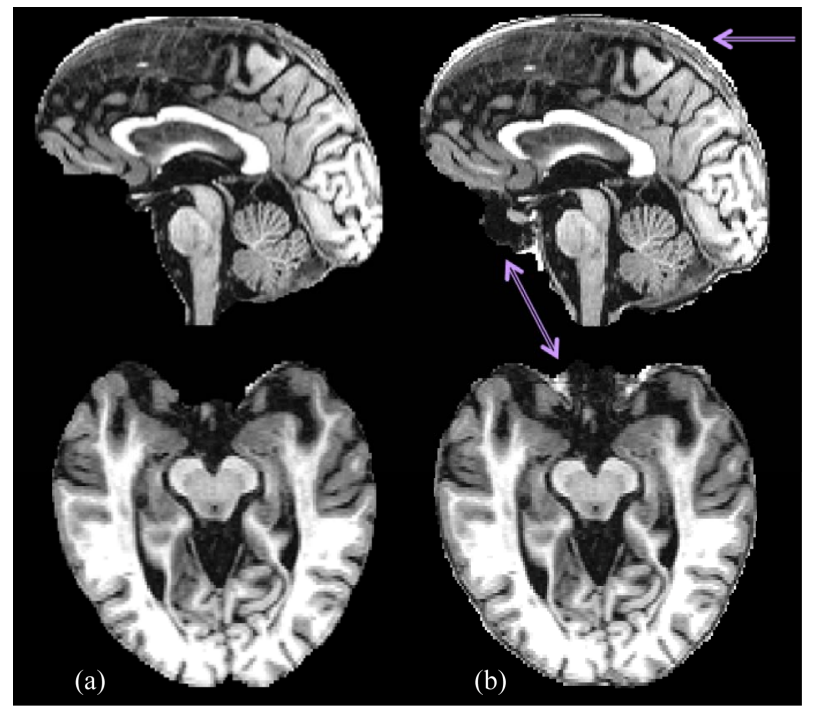

Figure 5. Sagittal and axial view of surrogate brain extraction of the same subject as Figure 3(a) using OET (a) and BET (b). The arrows indicate erroneously included areas.

ramen magnum only caused an insignificant trend in the difference in Bland-Altman analysis (v) (Figure 6. Positive slope of $\left.0.0031, R^{2}=0.0027\right)$.

\section{Discussions}

We compared a semi-automated method that uses colors for measuring ICV with a reference standard and two state-of-the-art automated methods. We found good agreement between the ICV measurements made by the reference standard traditional thresholding method followed by manual editing and the more automatic OET in Ana-

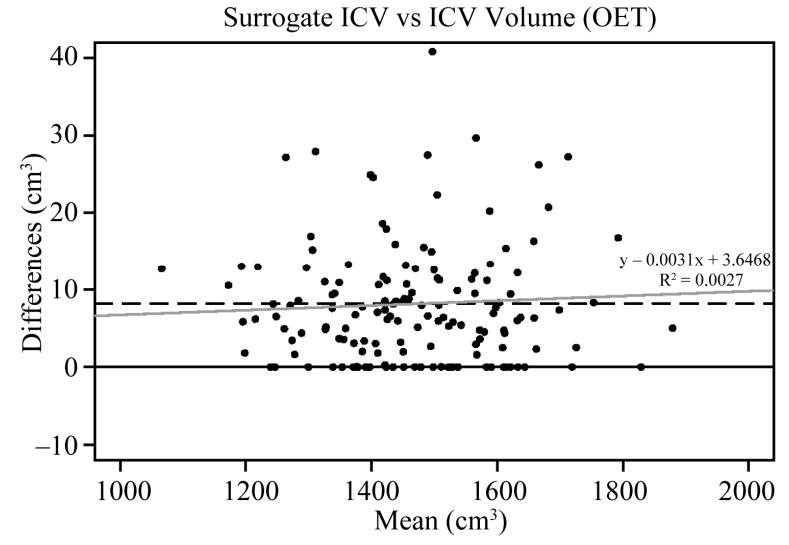

Figure 6. Bland-Altman showing the difference introduced to the surrogate ICV measure due to inclusion of the spinal cord below the foramen magnum.

lyze $^{\mathrm{TM}}$ in which morphological operations are added to the selection of the threshold to extract the ICV followed by minimal rectification of the ICV boundaries using MCMxxxVI to remove structures such as orbits, venous sinuses and other extracranial abnormalities and helping to identify the intracranial boundaries in difficult areas. This suggests that accurate measurement of ICV can be obtained faster using the latter method and this also reduces the amount of manual editing and hence observer dependence. The pituitary gland is a structure that causes controversy while delineating boundaries of the ICV. We found that the difference between including and excluding this structure was negligible and therefore there was no significant gain from spending time excluding this structure manually. Other fully automated methods were more variable, did not identify ICV accurately, and so should not be applied without visual inspection and a post-processing editing step; otherwise an error of up to $8.32 \%$ in the ICV may occur. Our results highlight the importance of using an accurate measure of ICV in establishing brain atrophy in healthy aging, showing that the difference in methodology alone could account for $5 \%-10 \%$ of the variance in a sample and therefore could mask subtle, but valuable, age-related changes.

The apparent contradiction of the automatic "surrogate ICV" by OET having a larger mean value than OET plus editing using MCMxxxVI is due to Bland Altman being unable to account for spatial disagreement. Visual inspection of the OET masks show the exclusion of the dural venous sinuses in some areas due to the automated threshold being higher than the threshold used for the reference standard. Thresholds are calculated using grayscale intensity levels. When a threshold that corresponds to a lower intensity level is set it will include more voxels in an image than the threshold corresponding to a high intensity level. For the Bland Altman analysis we 
subtracted the OET, OET plus editing using MCMxxxVI and BET volumes from the reference standard volumes and gave the percentage difference between methods. OET excludes intracranial structures in some areas but includes extracranial structures below the inferior boundary, when the extra step of removing the extracranial structures is taken the volume decreases causing the percentage difference to increase. Further analysis using a measure that provides information on spatial disagreement between methods would clarify these issues and highlight the importance of visually assessing segmentation output.

The strengths of our results include the use of a sample of brain images from older subjects selected to represent a range of cognitive and imaging factors and therefore to be biologically relevant. We had a large sample size which enabled us to detect quite small differences between measurement methods as well as any systematic bias. We tested semi-automated and automated ICV measurement methods, as well as the effect of including or excluding specific structures from the ICV. Another strength of our study is that while the reference standard was produced by an analyst well-trained in brain anatomy, the other techniques were applied by image analysts with strong technical background and minimal knowledge of brain anatomy, thus reproducing the common scenario in which some imaging research is carried out. Therefore, our results are generalisable. A limitation, however, is that although several approaches that combine thresholding with morphological operations for automated ICV measurement have been described, we only tested two methods of which one was programmed in a commercial software application and the other was a freely available academic package, both being considered to be representative of the available state-of-art techniques. We only calculated the agreement between techniques. It would be advisable to apply, in addition, other statistical measures that consider the defined similarity of the spatial information, like the Jaccard index, although this statistic does not inform on what differences might be due to.

While the inclusion of non-ICV tissue, such as the clivus and the sphenoid sinus, and the lack of having an anatomically-defined inferior limit to the intracranial cavity might not matter in younger normal people, it will introduce increasingly large systematic errors if used to measure ICV in older people in whom some degree of brain atrophy is virtually universal. Our results are in-line with previous studies that have attempted to improve BET for brain extraction, which have suggested including extra steps to remove non-brain structures [14]. Automatic methods still require visual assessment to ensure that extracranial structures are not included in the ICV and that true intracranial contents are not omitted, and a color fusion technique, like MCMxxxVI, freely available, can be extremely useful. Image processing techniques are improving all the time, but at present the methods tested in this work (which are representative of most available techniques) all require visual inspection and manual editing to derive the correct ICV and correct brain mask.

Many studies have demonstrated a variety of methods for producing color MR images [15,16,21-27], with evidence to suggest these techniques have advantages for the reporting clinical radiologist over the traditional greyscale images. Over the last two decades image fusion techniques utilising color overlay to display more than one parameter have become commonplace in clinical radiology. Examples include color Doppler flow displayed over greyscale structural ultrasound images, nuclear medicine studies such as octreoscan/PET overlaid in color over greyscale structural CT and fusion of structural ultrasound images (displayed in color) with corresponding CT or MRI cross-sectional imaging. However, as yet, the potential for combining more than one MRI sequence and displaying the co-registered images in color has not been exploited within clinical radiology.

The data presented here, along with a collection of related studies, suggests color fusion MRI techniques offer a valuable research tool. We would advocate the technique described here as a more reliable method of assessing ICV, particularly in older subjects and patients with neurological diseases with acknowledged damage on brain imaging. We would also highlight the considerable benefits color fusion MR techniques could offer to the clinical radiologist, and suggest such methods are more thoroughly evaluated within the clinical arena. We think it is time to give color a chance.

\section{Acknowledgements}

This work was funded by Age UK and the UK Medical Research Council as part of the Study Lothian Birth Cohort 1936, The Centre for Cognitive Aging and Cognitive Epidemiology (CCACE), The Row Fogo Charitable Trust and the Scottish Founding Council through SINAPSE collaboration. Funding (for CCACE; G0700704/ 84698) from the BBSRC, EPSRC, ESRC and MRC is gratefully acknowledged. The imaging was performed in the Brain Research Imaging Centre (www/sbirc.ac.uk), a SINAPSE Centre. The DICOM to analyze image format conversion tools used in the analysis were written by Dr. Paul A. Armitage.

\section{REFERENCES}

[1] J. S. Allen, J. Bruss, C. K. Brown and H. Damasio, "The Major Lobes and a Parcellation of the Temporal Region," Neurobiology of Aging, Vol. 26, No. 9, 2005, pp. 12451260. doi:10.1016/j.neurobiolaging.2005.05.023 
[2] D. D. Blatter, E. D. Bigler, S. D. Gale, S. C. Johnson, C. V. Anderson, B. M. Burnett, N. Parker, S. Kurth and S. D. Horn, "Quantitative Volumetric Analysis of Brain MR: Normative Database Spanning 5 Decades of Life," American Journal of Neuroradiology, Vol. 16, No. 2, 1995, pp. 241-251.

[3] M. A. Ikram, H. A. Vrooman, H. A. Vernooij, F. van der Lijn, A. Hofman, A. van der Lugt, W. J. Niessen and M. M. Breteler, "Brain Tissue Volumes in the General Elderly Population: The Rotterdam Scan Study," Neurobiology of Aging, Vol. 29, No. 6, 2008, pp. 882-890. doi:10.1016/j.neurobiolaging.2006.12.012

[4] R. I. Scahill, C. Frost, R. Jenkins, J. L. Whitwell, M. N. Rosser and N. C. Fox, "A Longitudinal Study of Brain Volume Changes in Normal Aging Using Serial Registered Magnetic Resonance Imaging," Archives of Neurology, Vol. 60, No. 7, 2003, pp. 989-994. doi:10.1001/archneur.60.7.989

[5] Y. Ge, R. I. Grossman, J. S. Babb, M. L. Rabin and D. L. Kolson, "Age-Related Total Gray Matter and White Matter Changes in Normal Adult Brain. Part I: Volumetric MR Imaging Analysis," American Journal of Neuroradiology, Vol. 23, No. 8, 2002, pp. 1327-1333.

[6] C. E. Coffey, G. Ratcliff, J. A. Saxton, N. R. Bryan, L. P. Fried and J. F. Lucke, "Cognitive Correlates of Human Brain Aging: A Quantitative Magnetic Resonance Imaging Investigation," Journal of Neuropsychiatry \& Clinical Neurosciences, Vol. 13, No. 4, 2001, pp. 471-485. doi:10.1176/appi.neuropsych.13.4.471

[7] R. T. Staff, A. D. Murray, I. J. Deary and L. J. Whalley, "Generality and Specificity in Cognitive Aging: A Volumetric Brain Analysis," Neuroimage, Vol. 30, No. 4, 2006, pp. 1433-1440.

doi:10.1016/j.neuroimage.2005.11.004

[8] G. Pengas, J. M. S. Pereira, G. B. Williams and P. J. Nestor, "Comparative Reliability of Total Intracranial Volume Estimation Methods and the Influence of Atrophy in a Longitudinal Semantic Dementia Cohort," Journal of Neuroimaging, Vol. 19, No. 1, 2009, pp. 37-46. doi:10.1111/j.1552-6569.2008.00246.x

[9] K. J. Ferguson, J. M. Wardlaw, C. L. Edmond, I. J. Deary, A. M. J. McLullich and N. C. Fox, "Intracranial Area: A Validation Method for Estimating Intracranial Volume," Journal of Neuroimaging, Vol. 15, No. 1, 2005, pp. 76-78. doi:10.1111/j.1552-6569.2005.tb00289.X

[10] R. N. K. Nandigam, Y. W. Chen, M. E. Gurol, J. Rosand, S. M. Greenberg and E. E. Smith, "Validation of Intracranial Area as a Surrogate Measure of Intracranial Volume When Using Clinical MRI," Journal of Neuroimaging, Vol. 17, No. 1, 2007, pp. 74-77. doi:10.1111/j.1552-6569.2006.00069.x

[11] J. M. Burns, B. B. Cronk, H. S. Anderson, J. E. Donelli, G. P. Thomas, A. Harsha, W. M. Brooks and R. H. Swerdlow, "Cardiorespiratory Fitness and Brain Atrophy in Early Alzheimer's Disease," Neurology, Vol. 71, No. 3, 2008, pp. 210-216. doi:10.1212/01.wnl.0000317094.86209.cb

[12] R. J. Thoma, M. Monnig, F. M. Hanlon, G. A. Miller, H. Petropoulos, A. R. Mayer, R. Yeo, M. Euler, P. Lysne, S.
N. Moses and J. M. Canive, "Hippocampus Volume and Episodic Memory in Schizophrenia," Journal of International Neuropsychological Society, Vol. 15, No. 2, 2009, pp. 182-195. doi:10.1017/S1355617709090225

[13] S. M. Smith, "Fast Robust Automated Brain Extraction," Human Brain Mapping, Vol. 17, No. 3, 2010, pp. 143155. doi:10.1002/hbm. 10062

[14] M. Battaglini, S. M. Smith, S. Brogi and N. De Stefano, "Enhanced Brain Extraction Improves the Accuracy of Brain Atrophy Estimation," Neuroimage, Vol. 40, No. 2, 2008, pp. 583-589. doi:10.1016/j.neuroimage.2007.10.067

[15] G. N. Holland and P. A. Botomley, "A Color Display Technique for NMR Imaging," Journal of Physics E: Scientific Instruments, Vol. 10, No. 7, 1977, pp. 14-16. doi:10.1088/0022-3735/10/7/014

[16] K. L. Weiss, S. O. Stiving, E. E. Herderick, J. F. Cornhill and D. W. Chakeres, "Hybrid Color MR Imaging Display," American Journal of Roentgenology, Vol. 149, No. 4, 1987, pp. 825-829.

[17] M. C. Valdes Hernandez, K. J. Ferguson, F. Chapell and J. M. Wardlaw, "New Multispectral Data Fusion Technique in MRI for White Matter Lesion Segmentation: Method and Comparison with Thresholding in FLAIR Images," European Radiology, Vol. 20, No. 7, 2010, pp. 16841891. doi:10.1007/s00330-010-1718-6

[18] I. J. Deary, A. J. Gow, M. D. Taylor, J. Corley, C. Brett, V. Wilson, H. Campbell, L. J. Whalley, P. M. Visscher, D. J. Porteous and J. M. Starr, "The Lothian Birth Cohort 1936: A Study to Examine Influences on Cognitive Aging from Age 11 to Age 70 and Beyond," Geriatrics, Vol. 7, 2007, pp. 28-33. doi:10.1186/1471-2318-7-28

[19] J. M. Wardlaw, M. E. Bastin, M. C. Valdes Hernandez, S. Munoz Maniega, N. A. Royle, Z. Morris, J. Clayden, E. Sandeman, E. Eadie, C. Murray, J. Starr and I. J. Deary, "Brain Aging, Cognition in Youth and Old Age, and Vascular Disease in the Lothian Birth Cohort 1936: Rationale, Design and Methodology of the Imaging Protocol," International Journal of Stroke, 2011, Article in Press.

[20] J. M. Bland and D. G. Altman, "Statistical Methods for Assessing Agreement between Two Methods of Clinical Measurement," Lancet, Vol. 327, No. 8476, 1986, pp. 307-310. doi:10.1016/S0140-6736(86)90837-8

[21] M. V. Vannier, R. L. Butterfield, D. Jordan, W. A. Murphy, R. G. Levit and M. Gado, "Multispectral Analysis of Magnetic Resonance Images," Radiology, Vol. 154, No. 1, 1985, pp. 221-224.

[22] W. E. Phillips, H. K. Brown, J. Bouza and R. E. Figueroa, "Neuroradiologic MR Applications with Multiparametric Color Composite Display," Magnetic Resonance Imaging, Vol. 14, No. 1, 1996, pp. 59-72. doi:10.1016/0730-725X(95)02043-S

[23] H. K. Brown, T. R. Hazelton and M. L. Silbiger, "Generation of Color Composites for Enhanced Tissue Differentiation in Magnetic Resonance Imaging of the Brain," American Journal of Anatomy, Vol. 192, No. 1, 1991, pp. 23-34. doi:10.1002/aja.1001920104

[24] H. K. Brown, T. R. Hazelton, J. V. Fiorica, A. K. Parsons, 
L. P. Clarke and M. L. Silbiger, "Composite and Classified Color Display in MR Imaging of the Female Pelvis," Magnetic Resonance Imaging, Vol. 10, No. 1, 1992, pp. 143-154. doi:10.1016/0730-725X(92)90384-C

[25] M. G. Wells, P. F. Sharp and A. N. Law, "Principles and Appraisal of Combined Images in NMR," Medical and Biological Engineering and Computing, Vol. 27, No. 3, 1989, pp. 277-280. doi:10.1007/BF02441485

[26] B. Alfano, A. Brunetti, A. Ciarmiello and M. Salvatore, "Simultaneous Display of Multiple MR Parameters with 'Quantitative Magnetic Color Imaging,," Journal of Computer Assisted Tomography, Vol. 16, No. 4, 1992, pp.
634-640. doi:10.1097/00004728-199207000-00025

[27] R. L. Kamman, G. P. Stomp and H. J. Berendsen, "Unified Multiple-Feature Color Display for MR Images," Magnetic Resonance in Medicine, Vol. 9, No. 2, 1989, pp. 53-55.

[28] H. K. Brown, T. R. Hazelton, A. K. Parsons, J. V. Fiorica, C. G. Berman and M. L. Silbiger, "PC-Based Multiparameter Full-Color Display for Tissue Segmentation in MRI of Adnexal Masses," Journal of Computer Assisted Tomography, Vol. 17, No. 6, 1993, pp. 993-1005. doi: 10.1097/00004728-199311000-00030 